\title{
Modelos de atención de salud en el Perú. Contextos e influencias.
}

Vallejos-Ragas $R^{1, a}$; Vilcahuaman-Bernaola $J^{1, b}$. Modelos de atención de salud en el Perú. Contextos e influencias. Rev Estomatol Herediana. 2013 Ene-Mar;23(1):44-9

\section{RESUMEN.}

El artículo describe el proceso de reforma y cambio de los modelos de atención de salud en el Perú, los contextos e influencias que enmarcan estas transformaciones, así como la descripción de los modelos financieros y conceptuales que determinan y explican el rumbo y limitaciones del modelo de salud elegido. Así mismo, se señalan las características de los modelos de salud: Modelo de Atención Integral de Salud (MAIS) y MAISFBC, como las diferencias, debilidades y posibles dificultades durante su implementación. Se hace hincapié en las dificultades ocasionadas durante el cambio de los Programas Nacionales de Salud verticales, por las Estrategias Sanitarias Nacionales, que afectaron el desempeño de las actividades de Inmunización, Tuberculosis y Salud bucal.

\section{Palabras Clave: SISTEMA DE SALUD, ATENCIÓN A LA SALUD, POLÍTICA DE SALUD.}

MODELS OF HEALTH CARE IN PERU. Contexts and influences

\section{ABSTRACT}

The article describes the process of reform and changing patterns of health care in Peru, contexts and influences that frame these transformations, and a description of financing and conceptual models that determine and explain the course and model limitations chosen health. Likewise, it identifies the characteristics of health models, MAIS and MAISFBC, as differences, weaknesses and possible difficulties during implementation. The emphasis is on the difficulties arising during the change of vertical National Health Programs for the National Health Strategies, which affected the performance of the activities of Immunization, Tuberculosis and oral health.

Key words: HEALTH SYSTEMS; HEALTH CARE; HEALTH POLICY.
Raúl Vallejos Ragas ${ }^{1, a}$
Jhonny Vilcahuaman
1,b

Facultad de Estomatología. Universidad Peruana Cayetano Heredia. Lima, Perú

a Magister en Estomatología.

Cirujano dentista. Diplomado de salud pública y odontología comunitaria.

\section{Correspondencia:}

Mg. Raúl Vallejos Ragas Jirón Emilio Althaus 121 of. 601- Lima 14, Perú

Teléfono: 4702575

E-mail: raul.vallejos@gmail.com

\section{Antecedentes}

A principios del 2000, en el Perú se inició un proceso de replanteamiento del modelo de atención en salud. Esta decisión estuvo influenciada por los distintos esquemas de "reformas" que se discutían en Latinoamérica - Fortalecimiento del rol rector de Ministerio de Salud, nuevos modelos de prestación de servicios, asignación de recursos públicos con eficiencia y equidad, mejora de la calidad de servicios, introducción de indicadores de desempeño, entre otros (1). Así como el agotamiento de los 16 programas de salud de características verticales y centralistas (tabla 1), que presentaban duplicidad de actividades, ineficiencia en el logro de resultados y confusión organizacional. Finalmente estos fueron desactivados en el 2001, por iniciativa del Banco Mundial y el Ministerio de Econo- mía preocupados por la duplicidad de gasto y las iniciativas desarrolladas regionalmente con enfoques de atención más amplios (2).

Los proyectos desarrollados en esos años, por las direcciones regionales de salud en colaboración con la cooperación internacional evidenciaron distintos esquemas de prestación de servicios, que se basaban en un abordaje "integral" tanto a personas como a familias, incluyendo mejoras en la organización así como en trabajo extramural Tablas 2 y 3.

Es importante señalar que este proceso de replanteamiento del modelo, se desarrolló teniendo un Sistema de Salud, definido como segmentado (Ministerio de Salud, Seguridad Social, Sanidad de las fuerzas armadas y policiales, y el sector privado: servicios municipales, iglesia católica, clínicas privadas, ONG), además de evidenciar un pobre desempeño en la evaluación realizada en el año 2000 por la OMS, ubicándose en el puesto 129 de 172 países considerados.

El Ministerio de Salud (MINSA), reconoce que el sistema de salud se desenvuelve en forma desorganizada, ante lo cual crea el 2001 del Sistema Nacional Coordinado y Descentralizado de Salud (SNCDS), que tenía como fin articular a los subsectores o componentes del sistema. En el año 2003, impulsa un nuevo Modelo de Atención de salud (La Salud Integral compromiso de todos - El modelo de Atención Integral en Salud MAIS) (6), que junto al SNCDS, responderían al "problema de la segmentación e irracionalidad del sector salud" (5). 
Finalmente, la promulgación de la ley de Bases de Descentralización $\left(\mathrm{N}^{\circ} 27783\right)$ (7), añadiría un factor más al complejo funcionamiento en el sector salud y por consiguiente a la viabilidad de la aplicación del nuevo modelo de salud.

\section{Modelos financieros $y$ concep- tuales}

Para comprender las bases de un modelo de atención en salud, es necesario identificar otros dos modelos, el de financiamiento y el conceptual, que orientan el desenvolvimiento del primero.

El modelo de financiamiento peruano se puede denominar como híbrido o segmentado, porque se obtiene de diversas fuentes: a) Recabados principalmente de impuestos, incluído el subsidio total del llamado Seguro Integral de Salud (SIS) (esquema Beveridge - Británico), b) Del enfoque basado en la seguridad social, financiado por el empleador (distinto al esquema de

Tabla 1. Programas nacionales de salud - MINSA - 1992. (4)

\begin{tabular}{|l|l|}
\hline \multicolumn{1}{|c|}{ DIRECCION RESPONSABLE } & \multicolumn{1}{|c|}{ PROGRAMAS VERTICALES } \\
\hline Dirección de Programa de Salud, Mujer y Niño & Salud Materno Perinatal \\
\cline { 2 - 2 } & Cáncer de Cuello Uterino \\
\cline { 2 - 2 } & Enfermedades Diarreicas Agudas \\
\cline { 2 - 2 } & Infecciones Respiratorias Agudas \\
\cline { 2 - 2 } & Crecimiento y Desarrollo \\
\cline { 2 - 2 } & Inmunizaciones \\
\hline \multirow{2}{*}{ Dirección de Programas Sociales } & Planificación Familiar \\
\cline { 2 - 2 } & Mujer y Desarrollo \\
\cline { 2 - 2 } & Adolescente y del Escolar \\
\hline \multirow{2}{*}{ Dirección de Programa Enfermedades } & Tuberculosis y Lepra \\
\hline \multirow{2}{*}{ Transmisibles } & Malaria y enfermedades Metaxénicas \\
\hline \multirow{2}{*}{ Dirección de Programas Especiales } & Control de Zoonosis \\
\hline & SIDA y Enfermedades Sexuales \\
\hline & Bocio y Cretinismo \\
\cline { 2 - 2 } & Salud Bucal \\
\cline { 2 - 2 } & Salud Mental \\
\hline
\end{tabular}

Tabla 2. Proyectos con diversos enfoques de prestación de servicios.

\begin{tabular}{l|l|}
\multicolumn{1}{c|}{ PROYECTOS } & \multicolumn{1}{c}{ CARACTERISTICA } \\
\hline $\begin{array}{l}\text { Proyecto APRISABAC (Convenio } \\
\text { Multilateral Perú-Holanda-Suiza } \\
\text { con la DISA Cajamarca). } 1999\end{array}$ & $\begin{array}{l}\text { Desarrollaron un conjunto de estructuras y funciones } \\
\text { que organizan a la oferta de la oferta para proveer aten- } \\
\text { ción de salud y lograr objetivos sanitarios - incluyen } \\
\text { las unidades de producción, los procesos productivos en } \\
\text { salud, los programas de Salud y los Servicios de Salud. }\end{array}$ \\
\hline Proyecto 2000 & $\begin{array}{l}\text { Acciones extramurales como parte del seguimiento de } \\
\text { la entrega de servicios materno infantiles. }\end{array}$ \\
\hline MINSA-Región San Martín. 1999 & Atención Integral del Niño \\
\hline UNI - Una Nueva Iniciativa. & Vigilancia Familiar. Paquete Básico de Servicios de \\
1999 & Salud \\
\hline Proyecto de Salud y Nutrición & Proyecto Salud y Nutrición Básica \\
Básica (PSNB). 2001 &
\end{tabular}

Bismarck - modelo Alemán, donde el financiamiento es tripartito: empleado, empleador y estado), c) Del enfoque privado lucrativo (seguros privados y gasto de bolsillo) donde la persona financia directamente su atención, siendo la de bolsillo la principal fuente de financiamiento en la actualidad (8) y d) Un último esquema al que denominaremos caritativo, el que se obtiene de colectas públicas durante todo el año, con el fin de atender diversas dolencias (cáncer, enfermedades del corazón, discapacidades, quemaduras, etc.) no cubiertas por el sistema de salud. Estas diferentes fuentes, generan inequidad o respuestas diferenciadas a los distintos grupos adscritos a los subsistemas, representadas con coberturas bajas, deficiente calidad y una precaria capacidad reguladora.

Con relación al modelo conceptual o paradigma, de cómo es entendida la salud, sus causas o factores que la afectan y por consecuencia la forma de abordarla, en el Perú, ésta ha atravesado varias etapas en las últimas décadas, empezando por el Modelo Biomédico, también llamado Biologicista o curativo, orientado a la enfermedad, centrándose en los cambios biológicos para explicar la salud y la enfermedad, siendo la curación y la rehabilitación sus formas de atenderla. Posteriormente se identifica que el proceso salud-enfermedad está relacionada a un conjunto de factores (biológicos, culturales, socioeconómicos y ambientales) que actúan durante un periodo determinado y que ocasionan finalmente la enfermedad, lo que derivó en el modelo preventivo.

Las acciones preventivas se sumaron a las actividades curativas previamente descritas. 
Luego del informe Lalonde (década del '70) y la Carta de Otawa (década del '80), se incorpora el concepto de Promoción de la Salud, que propone el desarrollo de estilos de vida saludable y la creación de ambientes favorables, como elementos prioritarios para que las personas y comunidades sean saludables. Esto propició un fuerte acento en esta área, sumándose así, la promoción de la salud a los dos modelos anteriores, pero no alcanzando la magnitud del concepto y relegándose solamente a intervenciones orientadas a la educación en salud. El concepto contemporáneo de la salud, denominado Producción Social en Salud, - Proceso mediante el cual la interacción de los actores sociales entre sí (todo individuo, colectividad u organización) y de éstos con su entorno, genera como resultado la salud que caracteriza a una población (9), aún tiene un desarrollo incipiente en el sector.

Modelo de atención, definida como la construcción social que sustenta una respuesta individual o colectiva a una o más necesidades de salud.

\section{Modelo de Atención Integral en Salud - MAIS (2003 - 2011)}

"La provisión continua y con calidad de una atención orientada hacia la promoción, prevención, recuperación y rehabilitación en salud, para las personas, en el contexto de su familia y comunidad".

El modelo fue propuesto reconociendo ocho problemas en la oferta de los servicios de salud, donde sobresalían:

a) La inequidad y deficiencias en la cobertura.

b) Fallas de solidaridad.

c) Desarticulación entre los niveles de atención. d) Ineficiencia e ineficacia del sistema de salud.

e) Deficientes competencias requeridas del recurso humano de los equipos de salud (6).

La propuesta de atención integral suponía intervenciones de promoción, prevención, recuperación y rehabilitación ofrecidas de manera "integrada", a personas de acuerdo a las etapas de vida, familias y comunidad.

Las intervenciones del modelo, describían tres tipos (Fig. 1):

Programas de Atención Integral: atendería las principales necesidades de salud, mediante la oferta de servicios y la elaboración de paquetes de atención.

\section{Estrategias Sanitarias Nacionales}

$y$ Regionales: acciones intersectoriales que se orientaría hacia la prevención de los problemas prioritarios de salud, en la búsqueda de impacto sobre los reales factores que dan origen a estos problemas. No ligada a la producción de servicios. Este tipo de intervención ocuparía el espacio de los Programas de Salud verticales desactivados.
Lineamientos Técnicos para la Generación de Comunidades y Entornos Saludables: Enmarcado dentro del enfoque de Promoción de la Salud, orientándose hacia el desarrollo de estilos de vida saludables y la construcción de una sociedad que ofrezca condiciones de vida digna.

Como parte del monitoreo del proceso de implementación, se debieron apreciar cambios importantes, como el incremento del financiamiento, la modificación de infraestructura, replanteamiento organizacional y nuevas prácticas, sin embargo, fueron escasas las modificaciones realizadas en este sentido.

En este proceso de cambio de modelo de atención, la desactivación de los programas de salud verticales, en el 2001, generaron un vacío funcional en las actividades vinculadas a cada programa, siendo las más afectadas las de Inmunizaciones y Tuberculosis, las que requerían un seguimiento permanente y continuo de sus atenciones. Recién en el 2004, parte de los programas fueron reactivados con las Estrategias Sanitarias del nuevo modelo de atención (Tabla 3).

Fig. 1. Configuración del Modelo de Atención de Salud - MAIS

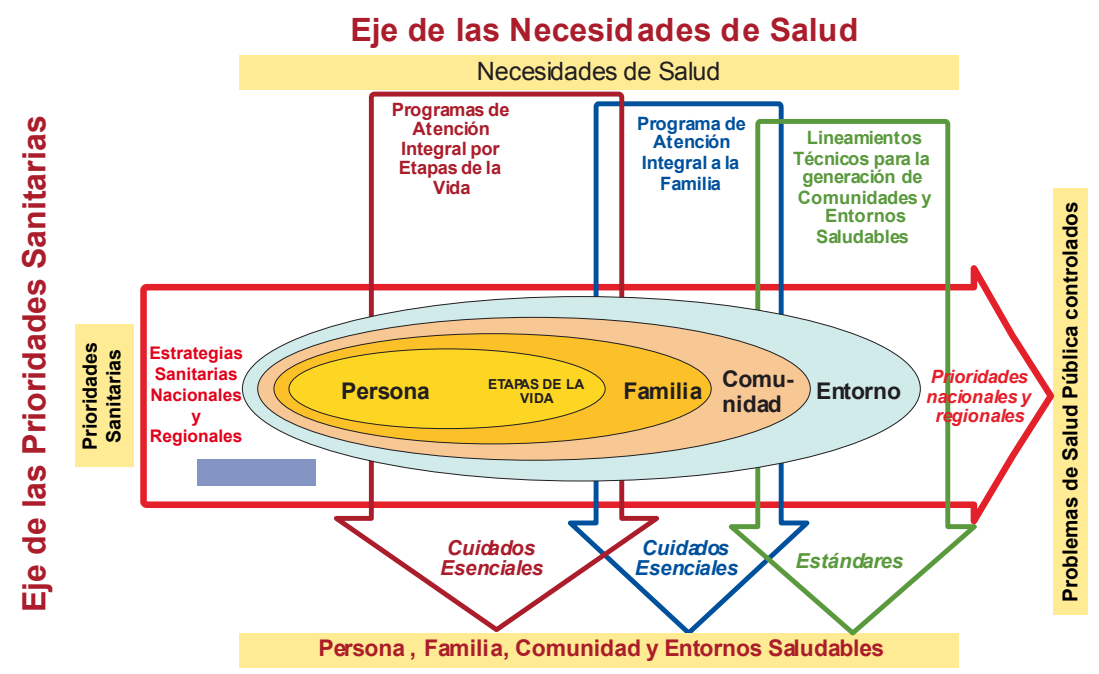


Tabla 3. Estrategias Sanitarias Nacionales de Salud - 2004.

\begin{tabular}{|c|c|}
\hline \multicolumn{2}{|r|}{ CREADAS EN EL 2004 (11 } \\
\hline DIRECCION RESPONSABLE & ESTRATEGIAS SANITARIAS \\
\hline \multirow[t]{6}{*}{ Dirección General de Salud de las Personas } & Inmunizaciones \\
\hline & Prevención y control de Enfermedades Metaxénicas y otras transmitidas por vectores. \\
\hline & Prevención y control de infecciones de transmisión sexual y VIH - SIDA. \\
\hline & Prevención y control de Tuberculosis. \\
\hline & Salud sexual y salud reproductiva. \\
\hline & Prevención y control de daños no transmisibles. \\
\hline Dirección General de Defensa Nacional & Accidentes de tránsito \\
\hline $\begin{array}{l}\text { Centro Nacional de Salud Intercultural del } \\
\text { Instituto Nacional de Salud }\end{array}$ & Salud de los pueblos indígenas \\
\hline $\begin{array}{l}\text { Centro Nacional de Alimentación y Nutri- } \\
\text { ción del Instituto Nacional de Salud }\end{array}$ & Alimentación y Nutrición Saludable \\
\hline $\begin{array}{l}\text { Dirección General de Promoción de la } \\
\text { Salud }\end{array}$ & Salud mental y cultura de Paz \\
\hline \multicolumn{2}{|r|}{ CREADAS POSTERIORMENTE } \\
\hline DIRECCION RESPONSABLE & ESTRATEGIAS SANITARIAS(Año de creación) \\
\hline \multirow[t]{5}{*}{ Dirección General de Salud de las Personas } & Salud bucal ( 2007)(12) \\
\hline & Salud ocular y prevención de la ceguera. (2007) (13) \\
\hline & $\begin{array}{l}\text { Atención a personas afectadas por contaminación por metales pesados y otras sustancias } \\
\text { químicas. (2008) (14) }\end{array}$ \\
\hline & Zoonosis (2008) (15) \\
\hline & Salud Familiar. (2009) (16) \\
\hline
\end{tabular}

Resaltan dos campos existentes como programas verticales - Salud Bucal y Zoonosis -, pero que no fueron considerados en las nuevas estrategias sanitarias. Siendo recién creados como estrategias sanitarias el 2007 y 2008 respectivamente.

El modelo nació con dificultades (sistema segmentado, financiamiento inequitativo, recurso humano con perfil biologicista (10) $\mathrm{y}$ sus acciones no abordaron los problemas descritos como base del fundamento del desarrollo de este modelo. Por lo cual, casi la totalidad de problemas se mantuvieron vigentes.

Un factor poco considerado, fue el recurso humano, elemento esencial para implementar un nuevo paradigma de salud. La ausencia de planificación y reformas que determinen, el perfil profesional, número, tipo y distribución no fue considerada. Dicha situación se evidencia entre otras cosas, en la dificultad de desarrollo de actividades fuera del contexto recuperativo y de rehabilitación en el MAIS.

En el 2011, el MINSA reconoce la presencia de casi la misma problemática descrita ocho años antes y propone realizar modificaciones al modelo a fin de que se "garantice a las personas, familia y comunidad el acceso a una atención integral de salud, oportuna y de calidad". Con dicho propósito, elabora y publica el Modelo de Atención Integral de Salud Basado en Familia y Comunidad (MAIS-FBC), que pretende actualizar los fundamentos y alcances de la atención integral de salud (17).
El contexto vigente en esa época, es influenciado por el enfoque de determinantes sociales, impulsado en el 2005, por la OMS mediante la formación de la Comisión sobre Determinantes Sociales de la Salud (18). Esta comisión, considera que "los factores estructurales y las circunstancias de vida, constituyen los determinantes sociales de la salud, que son la causa de la mayor parte de las desigualdades sanitarias entre los países y dentro de cada país".

Por otro lado, la OPS, plantea en el 2007, la "Renovación de la Atención Primaria de Salud en las Américas", señalando entre las justificaciones para esa estrategia, corregir las debilidades de algunos enfoques de la APS y reducir las inequidades en salud (19). 
La OPS, reconoce que muchos de los sistemas de salud de las Américas son segmentados, lo que genera dificultades de acceso, servicios de baja calidad técnica y el uso ineficiente de los recursos. Como respuesta a este problema, en octubre del 2009, lanza la iniciativa "Redes Integradas de Servicios de Salud" (RISS) (20).

Es así, que el MAIS-FBC, incorpora en su marco conceptual, a los Determinantes Sociales de la Salud, la Atención Primaria de Salud Renovada (APS-R) y las Redes Integradas de Servicios de Salud (RISS).

La implementación del nuevo modelo, planteaba desarrollarse con dos ejes básicos (Fig. 2):

\section{Eje de atención de las necesida-}

des: Respondería a las necesidades de salud, mediante paquetes de atención integral (actividades de prevención, promoción, recuperación y rehabilitación) a la persona y la familia. Incorporando también intervenciones a la comunidad del tipo promocional y preventivo.

\section{Eje de los determinantes sociales} de la salud: Que incluiría las intervenciones sobre los determinantes sociales de la salud, cuya responsabilidad estaría a cargo del gobierno nacional, gobierno regional y gobiernos locales.

Entre los cambios resaltantes de este modelo, se observa que las Estrategias Sanitarias Nacionales, no son parte de la configuración del MAIS-FBC; ni tampoco de los componentes de organización, prestación y financiamiento. Otro de los cambios que resalta es la agrupación de los Programas de Atención Inte- gral y los Lineamientos Técnicos del MAIS, en el denominado Eje de atención de las necesidades, siendo este último punto la única actividad de responsabilidad del MINSA, en este modelo (Tabla 4).

El documento técnico señalaba ciertas condiciones necesarias para que el MAIS-FBC alcance el éxito, proponiendo la elaboración de un Plan Nacional para su implementación, que incorpore:

a) El cambio de paradigma sobre la atención de salud.

b) El desarrollo de recursos humanos.

c) La reorientación del financiamiento.

d) Acciones intersectoriales y participación ciudadana. e) La articulación de las acciones de los gobiernos regionales y locales. Estas acciones como el plan nacional aún siguen pendientes.

\section{Reflexión Final}

La revisión y análisis de los modelos de salud en el Perú, buscan plasmar las razones y factores que determinan los cambios, su orientación y posibles problemas en su implementación.

La fragmentación del sistema de salud, es considerada, tanto para el MINSA, como para la OPS, como una de las causas principales de la ineficiencia e inequidad de la prestación de servicios de salud, sin embargo, esta ha sido abordada en los últimos años, utilizando distin-

Fig. 2. Configuración del Modelo de Atención Integral de Salud basado en familia y comunidad - MAIS-BFC

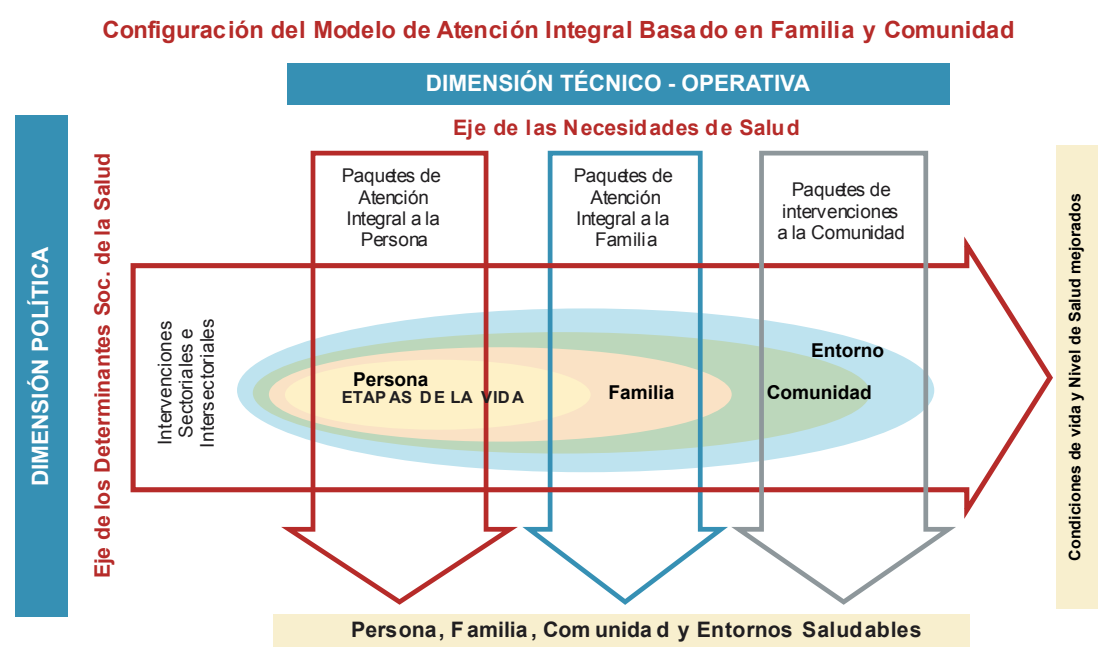

Tabla 4. Diferencias en los formas de intervención del Modelos de salud.

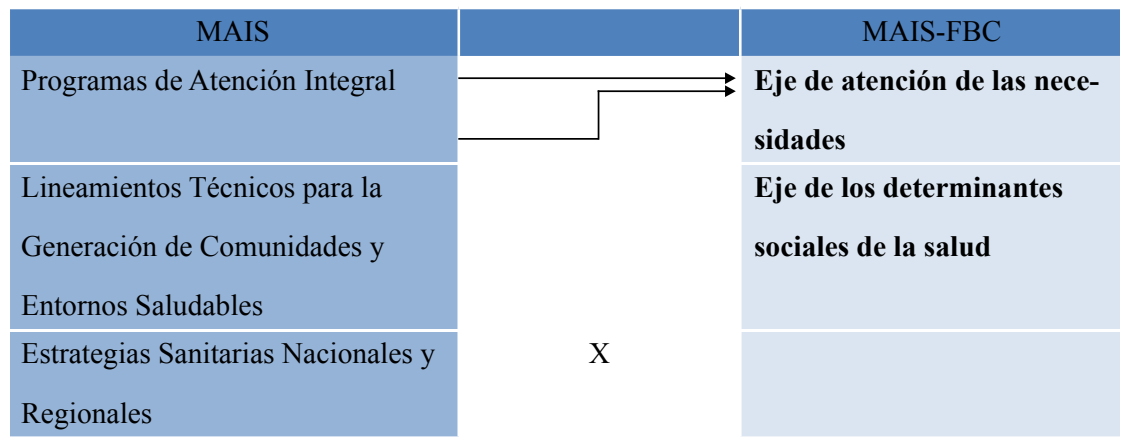


tos mecanismos, ya sea con la creación del Sistema Nacional Coordinado y Descentralizado de Salud (SNCDS),el desarrollo de Modelos de Atención (MAIS), la iniciativa de Redes Integradas de Servicios de Salud (RISS) y la Comisión Intergubernamental de Salud (CIGS) (21), las cuales no han logrado revertir estos problemas.

Es también evidente que la mayoría de iniciativas y políticas en el sector salud, han sido fuertemente influenciadas por agencias internacionales, sin tener el éxito esperado.

Finalmente, la propuesta de un modelo de salud en el Perú, deberá ir acompañado de la decisión política que permita las reformas estructurales del Sistema de Salud, su modelo de financiamiento y el cambio en la formación universitaria del paradigma en salud, que permita garantizar la salud de la población, mediante coberturas universales, equitativas y de calidad.

\section{Referencias Bibliográficas.}

1. Granados R. La reforma de los sistemas de salud: tendencias mundiales y efectos en latinoamérica y el caribe. Revista Gerencia y Políticas de Salud. 2002;1 (3):16-46.

2. Seminario L. Balance de la descentralización en la institucionalidad en salud. Lima: USAID/ Peru Health Office; 2011.

3. Nino A, Alcalde J, Meloni P. Modelo de atención. Aproximación Teórica. G\&D. Salud y Ambiente. 2008. (Citado el 12 de marzo del 2012) Disponible en:http://www.gycperu.com/ descargas/modelo $\% 20 \mathrm{de} \% 20$ atencion $\% 20 \% 20 \mathrm{~g} \& \mathrm{c} \% 20$.pdf.

4. Ministerio de Salud. Reglamento de organización y funciones del Ministerio de Salud. Decreto supremo $\mathrm{N}^{\circ}$ 002-92-SA. Lima: Ministerio de Salud; 1992.

5. Ministerio de Salud. La Salud Integral: Compromiso de todos Modelo de Atención Integral de Salud (MAIS), Lima-Perú 2003. Resolución Ministerial $N^{\circ} 729$ 2003 - MINSA. Lima: Ministerio de Salud.

6. Ministerio de Salud. Lineamientos de política sectorial para el período 2002 - 2012 y Principios Fundamentales para el Plan Estratégico Sectorial del Quinquenio Agosto 2001 - Julio 2006. Lima: Ministerio de Salud.

7. Congreso de la República. Ley de Bases de Descentralización (Ley $\mathrm{N}^{\circ}$ 27783). Lima, Perú Diario el Peruano;20 de julio del 2002.

8. Alcalde-Rabanal JE, Lazo-González O, Nigenda G. Sistema de salud de Perú. Salud Pública Mex. 2011;53 (S2): S243-S254.

9. Ministerio de Salud de Costa Rica. Modelo Conceptual y Estratégico de la Rectoría de la Producción Social de la Salud. 3ra edición. San José, Costa Rica: Ministerio de salud de Costa Rica; 2011.

10.Trentini M, Cubas MR. Ações de enfermagem em nefrologia: um referencial expandido além da concepção biologicista de saúde. Rev Bras Enferm. 2005 58(4):481-5.

11.Ministerio de Salud. Creación de las estrategias sanitarias Nacionales (RM 771-2004-MINSA). Lima, Perú: Ministerio de Salud; 2004.

12.Ministerio de Salud. Estrategia Sanitaria Nacional de salud bucal. RM. 649-2007. Lima, Perú: Ministerio de Salud; 2007.

13.Ministerio de Salud. Estrategia Sanitaria Nacional de salud ocular y prevención de la ceguera.
RM. 712-2007. Lima, Perú: Ministerio de Salud; 2007.

14.Ministerio de Salud. Estrategia Sanitaria Nacional de atención a personas afectadas por contaminación de metales pesados y otras sustancias químicas. RM. 425-2008. Lima, Perú: Ministerio de Salud; 2008.

15. Ministerio de Salud. Estrategia Sanitaria Nacional de Zoonosis. RM. 470-2008. Lima, Perú: Ministerio de Salud; 2008.

16.Ministerio de Salud. Estrategia Sanitaria Nacional de Salud Familiar. RM. 587-2009. Lima, Perú: Ministerio de Salud; 2009.

17.Ministerio de Salud. Modelo de Atención Integral de Salud Basado en Familia y Comunidad. Resolución Ministerial $N^{\circ} 464-$ 2011 - MINSA. Lima, Perú: Ministerio de Salud;2011.

18.Organización Mundial de la Salud. Comisión sobre Determinantes Sociales de la Salud. Informe final. Organización Mundial de la Salud; 2008.

19.Organización Panamericana de la Salud. Renovación de la Atención Primaria de Salud en las Américas. Organización Panamericana de la Salud; 2007.

20.Organización Panamericana de la Salud. Redes Integradas de Servicios de Salud: Conceptos, Opciones de Política y Hoja de Ruta para su Implementación en las Américas. Organización Panamericana de la Salud; 2010.

21.Instituto Suramericano de Gobierno en Salud. Sistemas de salud en Suramérica: desafíos para la universalidad la integralidad y la equidad. I. Rio de Janeiro: Instituto Suramericano de Gobierno en Salud; 2012.

Recibido : 03-02-2012

Aceptado: 29-05-2012 